\title{
Multiple-beam interference lithography with electron beam written gratings
}

\author{
H. H. Solak, ${ }^{\text {a) }}$ C. David, and J. Gobrecht \\ Laboratory for Micro and Nanotechnology, Paul Scherrer Institute, 5232 Villigen PSI, Switzerland \\ L. Wang and F. Cerrina \\ Department of Electrical and Computer Engineering, University of Wisconsin - Madison, \\ Madison, Wisconsin 53706
}

(Received 28 May 2002; accepted 9 September 2002)

\begin{abstract}
Interference lithography with multiple beams provides two-dimensional periodic patterns in a single exposure step. It is possible to obtain various symmetries and shapes such as sparse hole arrays or dots on a hexagonal grid. However, when the number of interfering beams exceeds three, the intensity pattern depends strongly on the relative phases of the beams. In this article we show that this problem can be overcome with a scheme where transmission diffraction gratings written on a single substrate create the interfering beams possessing the required phase relations. The relative phases of the diffracted beams are controlled by the relative positions of the gratings in the substrate plane. We used electron beam lithography with an interferometrically controlled stage to obtain the required precise placement of the gratings. This method enabled us to produce hexagonal and square arrays of holes where we used three and four beam configurations, respectively. Moreover, we created sparse hole arrays that have hexagonal and square symmetries with six and eight beam interference. The experiments were carried out in the extreme ultraviolet region with synchrotron radiation and with laser light in the visible spectrum. The technique requires spatially coherent light but it is achromatic and it is not sensitive to small errors in the alignment of the incoming beam. (C) 2002 American Vacuum Society. [DOI: 10.1116/1.1518015]
\end{abstract}

\section{INTRODUCTION}

Interference lithography (IL) is often used to produce large area periodic structures for research and production applications. Its ability to produce high-resolution patterns with relatively simple and low cost optics, as well as large depth of focus, makes it an attractive alternative to conventional lithography techniques. The technique has benefited greatly from the availability of highly coherent light from lasers operating in the visible and UV ranges. In the extreme ultraviolet (EUV) region, synchrotron sources have coherence properties that make them potential sources for IL applications that demand very high resolution. Synchrotron sources have been continuously improving over the last several decades in terms of coherence by tighter control of the electron beam dynamics. These improvements result in a smaller source size, emitting radiation in a narrow cone. The application of synchrotron radiation to IL was recognized early on, ${ }^{1,2}$ and several experiments have demonstrated the feasibility of the technique. ${ }^{3,4}$

In most applications of IL, two mutually coherent beams are brought together to form an interference fringe pattern, which is recorded in a photoresist film. In the two-beam interference method the intensity variation is sinusoidal and the resultant pattern is a grating with lines and spaces having roughly equal widths. In order to obtain other patterns, for example, a two-dimensional array of dots or a onedimensional sparse array of lines, one needs to add more beams into the mix either coherently or incoherently.

An example of incoherent addition is the commonly used

${ }^{a)}$ Electronic mail: harun.solakpsi.ch multiple exposure method to obtain a square array of dots or holes. In this method the sample is exposed with a two-beam interference pattern first, then the sample is rotated by $90^{\circ}$ and exposed with the same configuration of beams again. In this way one can obtain either holes or posts in the developed resist, which occur at the minima and maxima of the intensity profile, respectively, for a positive resist. The reverse patterns can be obtained by using a negative tone resist or an image reversal process. The intensity profile in this multiple exposure scheme places certain restrictions on the kinds of achievable patterns as well as the process latitude. In particular, the intensity distribution around the maxima is rather flat making it difficult to make the hole size substantially smaller than one half of the pattern period. ${ }^{5}$ Use of multiple beams (more than two) affords added flexibility in obtaining a desired intensity profile in interference lithography. ${ }^{5-8}$ Multiple beam interfence in combination with a multiple exposure scheme was described recently, where the standard two-beam interference as well as three, four, and five beam exposure techniques were evaluated in terms of process latitude and obtained resist profiles. ${ }^{6}$ In a later report Fernandex and Phillion pointed to the fact that in an interference scheme involving four or more beams the intensity profile depends dramatically on the relative phases of the interfering beams. ${ }^{8}$ They concluded that in a setup where the beams are directed to the exposure area by means of mirrors, it is necessary to control the mirror positions and alignments to a high degree of accuracy in order to satisfy and maintain the required phase relation. The alignment is also critical in order to have equal angles of incidences for all the beams, which is necessary to 


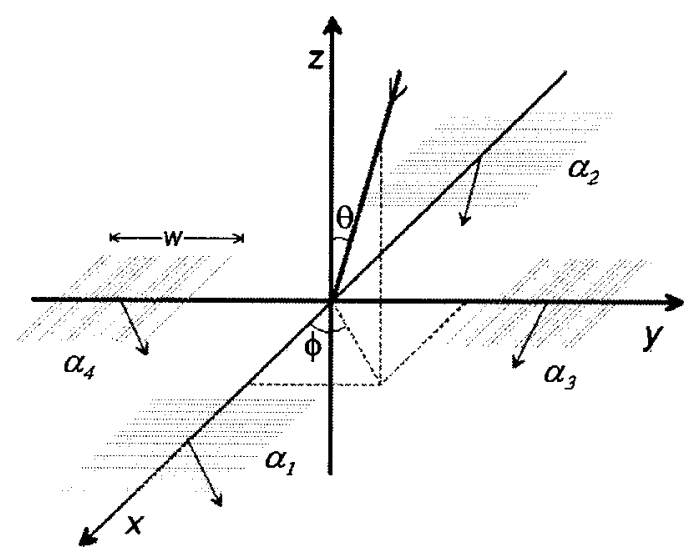

FIG. 1. Geometrical construction for the four-beam interference lithography. Four linear gratings lie in the $x y$ plane. The light is incident in the $-z$ direction, possibly making a small angle $\theta$ with the $z$ axis.

maintain the variance of the interference pattern in the direction perpendicular to the substrate.

In this article we show that we can effectively control the phase relations between the beams by a grating based multiple interference technique. The principle is illustrated in Fig. 1 where diffracted beams from transmission gratings written on a single mask overlap in a common area to yield the desired pattern. The required control of the phases of the beams is simply achieved by the control of the phases (i.e., positions) of the gratings on the mask. In practice we realized this by writing the diffraction gratings with an e-beam machine, which had an interferometrically controlled stage providing several nanometer placement accuracy. The use of multiple gratings on a mask for producing photonic crystals with hexagonal symmetry was reported recently. ${ }^{7}$ However, since the number of beams was under four in that study, the control of the beam phases was neither necessary nor mentioned.

\section{PHASE EFFECTS IN MULTIPLE BEAM INTERFERENCE}

As an example of the phase control achieved by this technique we will derive the intensity pattern for the case of four transmission gratings in the geometry defined in Fig. 1. We begin by writing the transmittance of a single grating as ${ }^{9}$

$$
T_{0}=\sum_{m} A m \exp \left(i \frac{2 m \pi}{p} x\right),
$$

where $p$ is the period of the grating. Transmission for a grating that is shifted by a distance $\Delta=p \alpha / 2 \pi$ in the direction perpendicular to the grating lines is then given by

$$
T_{0}=\sum_{m} A_{m} \exp \left(i \frac{2 m \pi}{p} x-i m \alpha\right) \text {. }
$$

Referring to the geometry in Fig. 1 we consider an incident plane wave making an angle $\theta$ with the $z$ axis on a mask that has four gratings lying in the $x y$ plane. We ignore the effects of different polarizations of the diffracted beams in order to emphasize the effect of the phases. This is a good approxi- mation when the diffraction angles are so small that the diffracted beams roughly have the same polarization. However, in the simulation and numerical results presented here the polarizations of the beams were always taken into account. The $m$ th diffracted order from the grating $G_{1}$ can be written as

$$
\begin{aligned}
E_{1 m}= & A_{m} \exp \left(i k \sin \theta \cos \phi x+i \frac{2 m \pi}{p} x\right. \\
& \left.+i k \sin \theta \sin \phi y-i m \alpha_{1}+i k_{z 1} z\right),
\end{aligned}
$$

where we have assumed an incident plane wave with unity amplitude. The term $k_{z 1}$ in Eq. (3) represents the $z$ component of the wave vector of the diffracted beam. To simplify the equations let us assume that the beam is normally incident on the grating, i.e., $\theta=0$. We analyze the interference field in the central overlap region where only four first-order diffracted beams given by

$$
E_{i}=A_{i} \exp \left(i \frac{2 \pi}{p} x-i \alpha_{i}+i k_{z i} z\right)
$$

are present. For a grating with symmetric groove profile the symmetric orders have equal amplitudes, i.e., $A_{m}=A_{-m}$. Moreover, for normal incidence and equal grating periods the $z$ components of the wave vectors are equal for all beams, i.e., $k_{z i}=k_{z}$. Adding amplitudes of all four beams and after some straightforward manipulation we obtain the total intensity as

$$
\begin{aligned}
I_{\text {total }}= & 2 A_{1}^{2}\left\{2+\cos \left(\frac{4 \pi}{p} x-2 \sigma_{x}\right)+\cos \left(\frac{4 \pi}{p} y-2 \sigma_{y}\right)\right. \\
& \left.+4 \cos \left(\frac{2 \pi}{p} x-\sigma_{x}\right) \cos \left(\frac{2 \pi}{p} x-\sigma_{y}\right) \cos \left(\delta_{x}-\delta_{y}\right)\right\},
\end{aligned}
$$

where we have introduced the terms $\sigma_{x}=\left(\alpha_{1}+\alpha_{2}\right) / 2, \sigma_{y}$ $=\left(\alpha_{3}+\alpha_{4}\right) / 2, \quad \delta_{x}=\left(\alpha_{1}-\alpha_{2}\right) / 2$, and $\delta_{y}=\left(\alpha_{3}-\alpha_{4}\right) / 2$. The last cosine term in Eq. (5) controls how the total intensity varies in space. This equation is equivalent to the results in Ref. 9, the difference being that here this controlling phase factor is determined by the phases of the four gratings on the mask.

There are two distinct intensity patterns one can obtain using the four-beam arrangement. One is equivalent to the incoherent addition of two crossed gratings, which occurs when the condition

$$
\delta_{x}-\delta_{y}=(n+1 / 2) \pi,
$$

where $n$ is an integer, is satisfied. A simulated pattern of this type is shown in Fig. 2(a). The other interesting intensity pattern is obtained when the above phase factor is equal to a multiple of $\pi$, i.e.,

$$
\delta_{x}-\delta_{y}=n \pi .
$$

In the latter case all four beams interfere constructively at points on a $45^{\circ}$ rotated square grid with a periodicity that is 


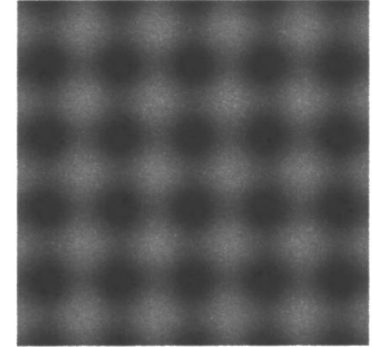

$\Phi=90^{\circ}$

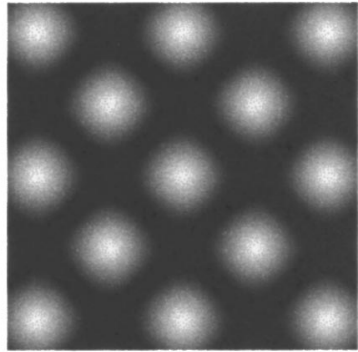

$\Phi=0^{\circ}$ (b)

FIG. 2. Simulated intensity patterns for the four-beam interference case for two values of the relative phase $\phi=\delta x-\delta y$. The periods of the square grids are $p / 2$ and $p / \sqrt{2}$, respectively.

$\sqrt{2}$ times greater than the previous pattern as shown in Fig. 2 (b). This configuration truly takes advantage of the coherent interference of four beams, creating an array of high intensity points, which are surrounded by areas of zero intensity.

\section{EXPERIMENT AND RESULTS}

Experiments with EUV light were performed on the EUV beamline at the Synchrotron Radiation Center of the University of Wisconsin. ${ }^{10}$ This undulator based beamline has a spherical grating monochromator which can reduce the spectral bandwidth to about $\Delta \lambda / \lambda=0.1 \%$. In this experiment a wider bandwidth was used in order to obtain higher flux since the interferometer does not require temporal coherence. Chemically amplified photoresist UV-6 (Shipley Co.) with a thickness of $120 \mathrm{~nm}$ was exposed using a configuration of four gratings like the one in Fig. 1. The phases of the gratings were designed to satisfy the relation in Eq. (7). Figure 3 shows the array of holes with a period of $141 \mathrm{~nm}$ printed with this arrangement.

The EUV transmission gratings were fabricated on 100nm-thick $\mathrm{Si}_{3} \mathrm{~N}_{4}$ membranes formed on $\mathrm{Si}$ wafers. A Cr film with a thickness in the $40-70 \mathrm{~nm}$ range was deposited onto the membranes by thermal evaporation. The samples were then spin coated with 70-nm-thick poly(methylmethacrylate) (PMMA) films. The grating structures were exposed with a LION-LV1 e-beam system (Leica Microsystems, Jena) using

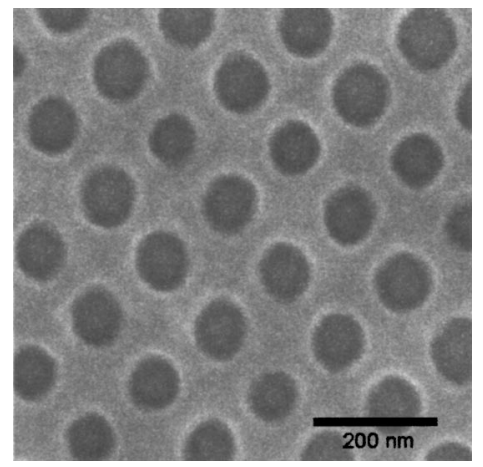

FIG. 3. Array of holes printed in photoresist with four-beam interference with EUV illumination. The period is $141 \mathrm{~nm}$.

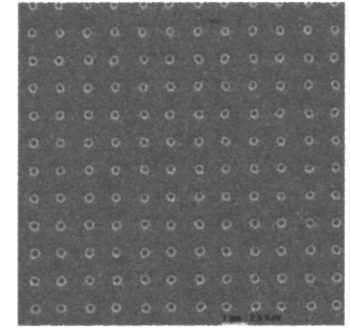

(a)

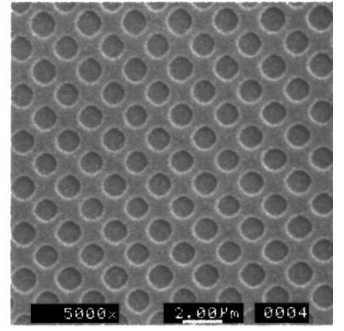

(b)
FIG. 4. Arrays of holes printed in photoresist with four-beam interference and laser illumination. The grating masks had same periodicity in the two cases but the gratings were placed so as to obtain phase factors ( $\phi=\delta x$ $-\delta y$ ) $90^{\circ}$ and $0^{\circ}$ in (a) and (b), respectively. The patterns are directly comparable with the simulation results presented in Fig. 2.

continuous path control mode. The system has an interferometer-controlled stage with several nanometer scale accuracy, which ensured precise control of the placement, i.e., phase, of these high-resolution gratings. The developed patterns were transferred into the Cr layer by a dry etching process.

In the second set of experiments a $\mathrm{HeCd}$ laser operating at $442 \mathrm{~nm}$ was used to expose interference patterns. The setup included a pinhole spatial filter to illuminate the gratings with a high degree of coherence. The diffraction gratings were patterned on standard glass mask substrates. As in the EUV case, the gratings were written with e-beam lithography and the pattern was transferred into a Cr layer with dry etching. The $\mathrm{Cr}$ film was thick enough to completely block the transmission of light; therefore the gratings were of pure absorption type. For improved efficiency one can choose to use phase or blazed-type gratings. The IL experiments were all performed with large enough grating periods so that the angle of diffraction, $\theta=\sin ^{-1}(\lambda / p)$ was always less than $27^{\circ}$. This is necessary to avoid modulation-degrading effects of polarization when the beams meet each other at larger angles. We measured the TE and TM diffraction efficiency to be roughly the same for all of the gratings, which ensured that the interfering beam had equal intensities.

Multi-beam interference patterns obtained with this system were recorded in commercial photoresist Microposit 1805 (Shipley Co). Substrate reflection was largely eliminated by a $\mathrm{ZnS}$ dielectric anti-reflection film deposited on the substrates before photoresist coating. Figures 4(a) and 4(b) show two arrays exposed with two very similar grating designs. The only difference was the relative phase factor $\phi$, which was equal to $\pi / 2$ and 0 for the two cases, respectively. These resist patters are directly comparable and in good agreement with the simulated intensity maps in Fig. 2. Experiments with other geometries included three-, six-, and eight-beam configurations. In the three-beam case gratings were arranged on the sides of an equilateral triangle so that the diffracted beams overlapped within the central triangular area. In this case the two-dimensional intensity pattern is independent of the phases of the beams except for mere shifts. Figure 5 shows a pattern obtained in this way. 


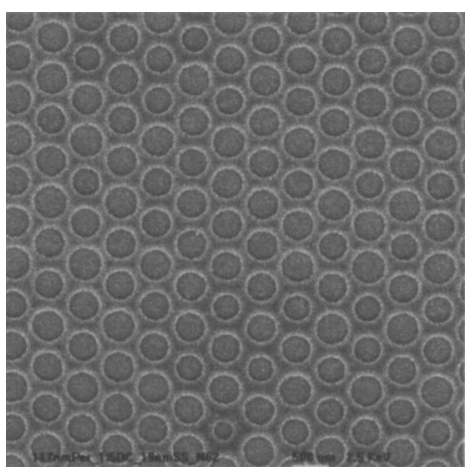

FIG. 5. Hexagonal array of holes created with three-beam interference. The nearest neighbor distance is $2 p / 3$, where $p$ is the diffraction grating period.

Six- and eight-beam configurations can be considered as extensions of the three- and four-beam cases. In both cases an inner set of gratings with a period $p$ create a low frequency pattern in the exposure plane. An outer set of gratings with half the period, i.e., $p / 2$, diffract the beams into the same overlap area as the first set. Thus the second set creates a similar intensity pattern in the same area with double the frequency. The phases of the first and second sets are matched so that the two patterns add up in phase resulting in cancellation of the peaks at alternate positions. The result is a two-dimensional array of intensity peaks separated by a large distance in between, i.e., a sparse array. Example patterns are seen in Fig. 6 as well as the simulated intensities. The residual intensity structure in the areas between the holes is seen both in simulation results as well as resist patterns as a surface relief structure in the resist.

When the diffracted beams make different angles with the normal then the invariance of the intensity pattern in the $z$ direction is lost. Instead one gets a periodically varying in-

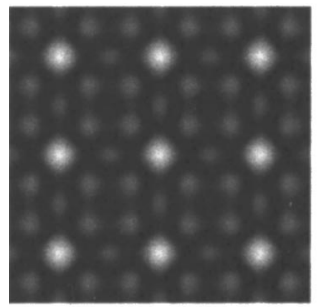

(a)

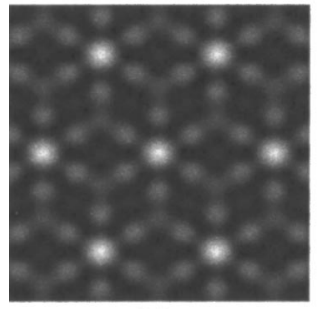

(c)

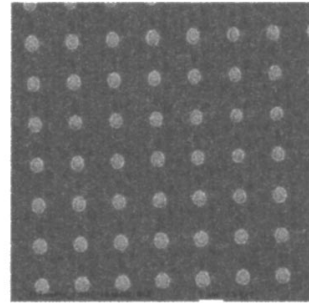

(b)

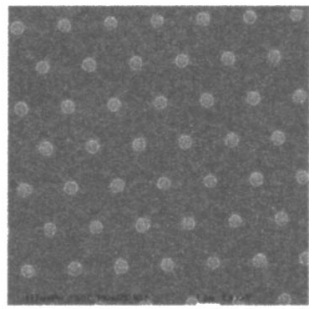

(d)
FIG. 6. Simulation and experimental results for the eight (a), (b), and six (c), (d) beam interference configurations. Note that the remaining intensity in the cancelled peaks are visible in the simulation pictures. Simulation and experimental results are not to the same scale. tensity pattern along this direction. This is the case for the six- and eight-beam configurations described above. Therefore it is necessary to position the substrate parallel to the mask and place it at one of the periodic distances where the desired intensity profile is obtained. The depth of focus around these periodic positions is close to the familiar formula for depth of focus in imaging systems, i.e., DOF $=\lambda / \mathrm{NA}^{2}$, where NA is the numerical aperture for the beams making the largest angle with the normal.

Another concern in IL with multiple beam is the sensitivity to errors in the alignment of the incoming beam. ${ }^{8}$ This can change the phase relations between the interfering beams across the field of exposure and thus cause moiré-like variations in the printed pattern. The misalignment tolerance in the angle of incidence is given by the relation

$$
\theta \ll \frac{p}{\sqrt{z \lambda}},
$$

where $\theta$ is as defined in Fig. 1 and $z$ is the distance between the gratings and the exposure plane. Derivation of this relation will be presented elsewhere. For an EUV experiment performed at $\lambda=13.4 \mathrm{~nm}, p=100 \mathrm{~nm}, z=1 \mathrm{~mm}$, we get a very comfortable misalignment margin of about $20 \mathrm{mrad}$.

\section{SUMMARY}

Two-dimensional periodic patterns are desirable in a variety of applications. While multiple beam IL has the potential for creating such patterns, it is hampered by the requirement of phase control between the interfering beams. In this study we have demonstrated with analytical derivation, simulations, as well as experimental results that transmission diffraction gratings written on a single substrate can be used to control the relative phases between the beams. The required placement accuracy of the gratings is easily satisfied by modern electron beam lithography machines. For preserving the coherence of the gratings over large distances for large area applications we envision using a two-beam IL to create the diffraction gratings.

The experiments were performed at EUV and visible wavelengths. Undulators at modern synchrotron radiation facilities emit EUV light with high spatial coherence. ${ }^{11}$ Since the technique is achromatic, the finite temporal coherence of the undulator EUV source did not limit the modulation or the number of fringes obtained in our experiments. Therefore one can fully take advantage of the short wavelengths available from undulator sources, without being limited by the relatively wide spectrum of their light compared to lasers.

\section{ACKNOWLEDGMENTS}

The authors thank S. Stutz, D. Bächle, B. Haas, L. Heyderman, and H. Schift from the Laboratory for Micro and Nanotechnology at the Paul Scherrer Institut, and J. Wallace and A. Ho from the Center for NanoTechnology of the University of Wisconsin-Madison for their help with the experiments and useful discussions. 
${ }^{1}$ R. Thatchyn, E. Kallne, A. Toor, T. Cremer, and P. Csonka, Rev. Sci. Instrum. 60, 1579 (1989).

${ }^{2}$ A. Yen, M. L. Schattenburg, and H. I. Smith, Appl. Opt. 31, 2972 (1992).

${ }^{3}$ M. Wei, D. T. Attwood, and T. K. Gustafson, J. Vac. Sci. Technol. B 12, 3648 (1994).

${ }^{4}$ H. H. Solak, D. He, W. Li, S. Singh-Gasson, F. Cerrina, B. H. Sohn, X. M. Yang, and P. Nealey, Appl. Phys. Lett. 75, 2328 (1999).

${ }^{5}$ A. Fernandez, J. Y. Decker, S. M. Herman, D. W. Phillion, D. W. Sweeney, and M. D. Perry, J. Vac. Sci. Technol. B 15, 2439 (1997).

${ }^{6}$ X. Chen, S. H. Zaidi, S. R. Brueck, and D. J. Devine, J. Vac. Sci. Technol. B 14, 3339 (1996).
${ }^{7}$ I. B. Divliansky, A. Shishido, I. Khoo, T. S. Mayer, D. Pena, S. Nishimura, C. D. Keating, and T. E. Mallouk, Appl. Phys. Lett. 79, 3392 (2001).

${ }^{8}$ A. Fernandez and D. W. Phillion, Appl. Opt. 37, 473 (1998).

${ }^{9}$ A. Yen, E. H. Anderson, R. A. Ghanbari, M. L. Schattenburg, and H. I. Smith, Appl. Opt. 31, 4540 (1992).

${ }^{10}$ H. H. Solak, W. Li, D. He, J. Wallace, and F. Cerrina, AIP Conf. Proc. 521, 99 (2000).

${ }^{11}$ D. T. Attwood, P. Naulleau, K. A. Goldberg, E. Tejnil, C. Chang, R. Beguiristain, P. Batson, J. Bokor, E. M. Gullikson, M. Koike, H. Medecki, and J. H. Underwood, IEEE J. Quantum Electron. 35, 709 (1999). 